\title{
A robust multi-objective production planning
}

\author{
Mohsen Gharakhani $^{\mathrm{a} *}$, TahereTaghipour ${ }^{\mathrm{b}}$ and Kambiz Jalali Farahani ${ }^{\mathrm{b}}$ \\ ${ }^{a}$ Department of Industrial Engineering, Sharif University of Technology, Tehran Iran \\ ${ }^{b}$ Department of Management, Islamic Azad University, South branch, Tehran, Iran

ART I CLEINFO ABSTRACT

Article history:

Received 1 January 2010

Received in revised form

10 March 2010

Accepted 1 April 2010

Available online 18 April 2010

Keywords:

Production planning

Robust optimization

Reliability

Redundancy

Multi-objective

\begin{abstract}
When a production facility is designed, there are various parameters affecting the number machines such as production capacity and reliability. It is often a tedious task to optimize different objectives, simultaneously. The other issue is the uncertainty in many design parameters which makes it difficult to reach a desirable solution. In this paper, we present a new mathematical model with two objectives. The primary objective function is considered to be the production capacity and the secondary objective function is total reliability. The proposed model is formulated on different units of production which are connected together in serial form and for each unit, we may have various machines. The resulted model is formulated using recent advances of robust optimization and solution procedure is analyzed with some numerical examples.
\end{abstract}

\section{Introduction}

During the past two decades, there have been tremendous efforts to provide comprehensive model which could consider various parameters affecting the optimal planning. One of the primary issues is the uncertainty which exists among input parameters. There are also many real-world applications where a small change in the input data could change the optimal solution, significantly. In fact, one may lose the feasibility when there is a small change on the input data. Soyester (1973) is believed to be the first one who addresses the uncertainty in the context of the linear programming. For over two decades, his idea was on the literature but practically no one used it since the implementation could result a very conservative optimal solution. Ben-Tal and Nemirovski (2000) develop a new robust optimization based on the adaptation of cone programming. The new method turns an ordinary linear programming problem with uncertain data into a new form where the optimal solution lies inside a counter-part. Therefore, we may lose part of the optimality but the final solution remains feasible whenever there are changes on the input data. They also show, in their work, that many engineering benchmark problems could end to an infeasible solution when some small changes occur on input data and their method could immune the final solution even if all input parameters are perturbed. Although, Ben-Tal and Nemirovski's robust method has proven to be efficient but it needs to use non-linear optimization techniques which is not popular among many practitioners. Bertsimas and Sim (2003) propose a new robust optimization technique where the robust technique does not need to change the structure of the original problem and the quality of the final robust solution could compete with the other techniques. Sadjadi and Omrani (2004) propose a robust data envelopment analysis and compare the final solutions resulted from both Bertsimas and Ben-Tal and report no significant difference between these two methods. Shafia et al. (2010) adapt the Bertsimas and Sim's robust techniques on a mixed integer train

* Corresponding author. Tel./fax: +98-912-5939771.

E-mail addresses: m.gharakhani@sharif.edu (M. Gharakhani), 
formation and report promising results. Nikoofal and sadjadi (2010) use the same robust technique for $p$ median problem and report that the new robust model could handle the uncertainty on negligible cost. The other issue of many facility design problem is to handle different objectives at the same time. In fact, in many problems, one may face criteria which are in conflict. Therefore, we may use multiple objective decision techniques to find an efficient solution (sadjadi, et al. (2009)). When a production facility is designed, one may consider total capacity as a primary objective which leads to higher profitability. However, an important aspect of designing a good production facility is also to minimize the total reliability. In other word, when there is unit with relatively low reliability, we may also expect a significant interruption cost at any time which could affect profitability as well. Sadjadi and Soltani (2009) present a new method to find the near optimal of a redundancy problem in a series-parallel machines. Their method considers a general problem where there are many constraints involved and a meta-heuristic method is proposed to solve such problem. The implementation of meta-heuristic approaches for redundancy problems has become popular since the complexity of many such problems is NP-Hard (Ramirez-Marquez 2004). An integration of reliability and production planning has recently gained much attention among many practitioners (Khademi 2006, Kenné, 2007). In this paper, we propose a new multi-objective production planning where the numbers of facilities are located in series-parallel operations. In other word, we consider a production system where a part needs to pass different production units in serial sequence. For each unit the production capacity is limited, so the total production capacity is determined by the minimum capacity. On the other hand, the reliability of the system is also mainly determined by the unit with minimum reliability. We consider two objectives which are in conflict: The first objective is to maximize the capacity with the aim of maximizing the profitability and the second objective is to maximize the reliability which may indirectly increase the profitability. The proposed model of this paper also deals with two separate constraints: The first sets are mainly related to factory conditions such as space, time, etc which are subject to uncertainty and the second constraint is final budget which would be devoted to production plan to increase the total reliability. This paper is organized as follows. We first present the problem statement and the necessary notations in section 2 . In section 3 , we present the robust proposed model and the implementation of the proposed model is demonstrated using some numerical examples. We also discuss the results and the details of the implementation of our proposed method. Finally, conclusion remarks are given at the end to summarize the contribution of the paper.

\section{Problem Statement}

Consider a production facility with $s$ different departments which are connected together in serial form. Fig 1 shows the structure of our proposed model.

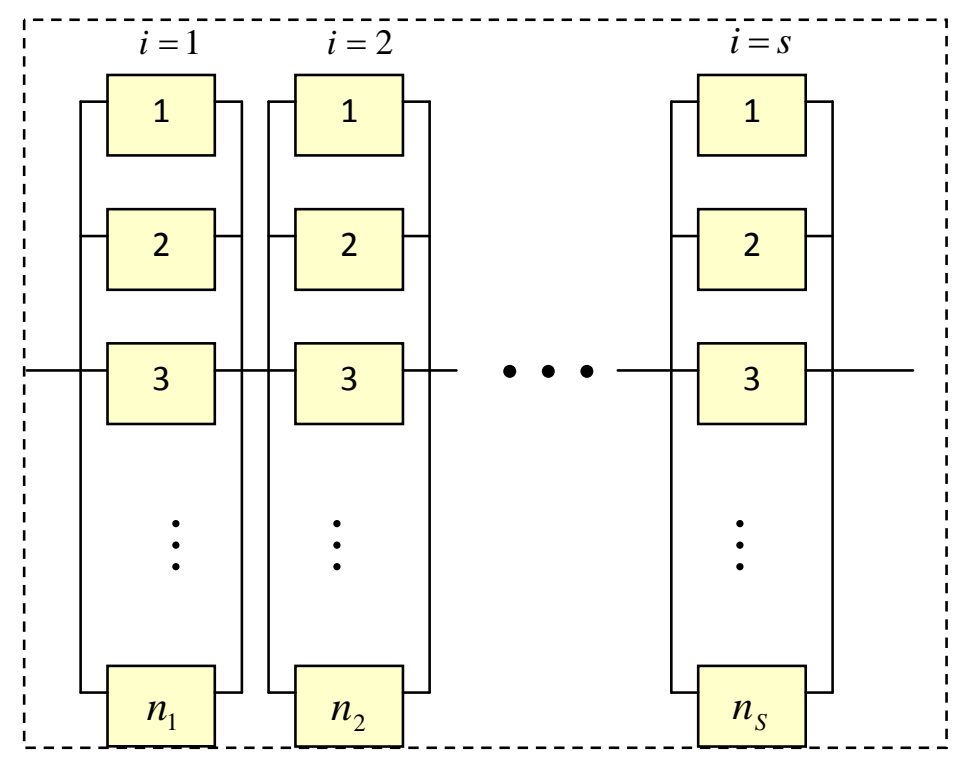

Fig.1.The serial-parallel system

Let $p_{i}$ and $x_{i}$ be the production capacity and the number of facilities of each unit production $i$, respectively. For the sake of simplicity, we assume all production facilities located in each cell are unique with equal 
reliability. Let $A$ and $b$ be the constraints associated with some restrictions such as space, price, etc. Model (1) represents the problem formulation with an objective of maximizing the minimum number of production capacity.

$$
\begin{array}{cc}
\max & \min \left\{p_{i} x_{i}\right\} \\
\text { s.t. } & A x \leq b, \\
& x_{i} \geq 1,2, \cdots .
\end{array}
$$

Since the objective function of (1) is in a form of max-min we use an auxiliary variable $w$ which yields the following,

$$
\min \left\{w \mid p_{i} x_{i} \geq w, A x \leq b, l \leq x \leq u, x_{i} \geq 1,2, \ldots, w \geq 0\right\}
$$

Problem (2) is a mixed integer problem which could be solved using any traditional mixed integer algorithms. We also add some lower and upper bounds for all variables to have practical solutions. The primary concern is that production capacity of each unit $\left(p_{i}\right)$ is normally under uncertainty. Let $\tilde{p}_{i}$ be the uncertain capacity where it is located in a symmetric interval of $\left[p_{i}-\hat{p}_{i}, p_{i}+\hat{p}_{i}\right]$ where $p_{i}$ indicates the nominal value for $\tilde{p}_{i}$, i.e. it follows a uniform distribution which incorporates the uncertain situations. The amount of $\hat{p}_{i}$ is the maximum possible deviation of both negative and positive values from nominal value. Since it is very unlikely that all uncertain parameters get their worst case limit, Bertsimas and Sim (2003) introduce a parameter $\Gamma_{i}$ with $0 \leq \Gamma_{i} \leq\left|J_{i}\right|$ to adjust the conservatism level of the optimal solution. In practice, only a few coefficients are subject to change. Therefore model (2) can be formulated in robust form using Bertsimas and Sim [3] in the following form,

$$
\max \left\{w \mid w-p_{i} x_{i}+z_{i} \Gamma_{i}+q_{i} \leq 0, z_{i}+q_{i} \geq \hat{p}_{i} x_{i}, A x \leq b, x_{i} \geq 1,2, \cdots, w, z_{i}, q_{i} \geq 0 .\right\}
$$

Problem (3) is a mixed integer program with additional variables of $z_{i}$ and $q_{i}$ where they are associated with the uncertain constraints and variables, respectively. Next section, we demonstrate the implementation of the proposed method using a numerical example.

\section{Example 1.}

Consider the following data set,

$$
s=5, p_{1}=40, p_{2}=10, p_{3}=30, p_{4}=20, p_{5}=45, A=\left[\begin{array}{lllll}
3 & 4 & 3 & 2 & 5 \\
2 & 3 & 4 & 5 & 4 \\
5 & 2 & 5 & 7 & 3
\end{array}\right], b=\left[\begin{array}{l}
50 \\
60 \\
70
\end{array}\right] \text {. }
$$

Problem (3) is solved using different uncertainty for all variables. When $\Gamma_{i}=0$, there is no uncertainty associated with $p_{i}$ and the optimal solution for (2) and (3) are the same. As $\Gamma_{i}$ increases, the uncertainty appears for all capacities and we get more conservative solutions. As we can observe from Table (1), the optimal solutions are more conservative when the budget of uncertainty for all parameters increases. The other observation is that the order of optimal facility allocation changes as the budget of uncertainty increases. In other word, when $\Gamma_{i}=0.1$, there are only two facilities allocated to the first department and five facilities are assigned to the second department. However, when $\Gamma_{i}=0.9$ the number of optimal facilities is reduced to one for the first department and this number is increased to seven for the second 
department. The last column of the table also demonstrates the total production capacity. As we can see from Table 1, this number is reduced from 50 to 40 when we increase the budget of uncertainty.

\section{Table 1}

The optimal solution with uncertain parameters

\begin{tabular}{cccccccc}
\hline Variable & $x_{1}$ & $x_{2}$ & $x_{3}$ & $x_{4}$ & $x_{5}$ & Total production Capacity \\
\hline$\hat{p}_{i}$ & 0.1 & 0.5 & 0.2 & 0.3 & 0.25 & \\
$\Gamma_{i}=0$ & 2 & 5 & 2 & 4 & 2 & 50 \\
$\Gamma_{i}=0.1$ & 2 & 5 & 2 & 4 & 2 & 50 \\
$\Gamma_{i}=0.2$ & 2 & 5 & 2 & 4 & 2 & 50 \\
$\Gamma_{i}=0.3$ & 2 & 5 & 2 & 3 & 2 & 50 \\
$\Gamma_{i}=0.4$ & 2 & 6 & 2 & 3 & 1 & 45 \\
$\Gamma_{i}=0.5$ & 2 & 6 & 2 & 3 & 1 & 45 \\
$\Gamma_{i}=0.6$ & 2 & 6 & 2 & 4 & 1 & 45 \\
$\Gamma_{i}=0.7$ & 1 & 6 & 2 & 3 & 2 & 40 \\
$\Gamma_{i}=0.8$ & 1 & 7 & 2 & 3 & 1 & 40 \\
$\Gamma_{i}=0.9$ & 1 & 7 & 2 & 3 & 1 & 40 \\
$\Gamma_{i}=1$ & 1 & 7 & 2 & 3 & 1 & 40 \\
\hline
\end{tabular}

\section{A Multi-objective model}

The primary objective of problem (3) is to increase the output production. However, one may argue that in case there is an interruption in any production facilities due to failure of one or more facilities, we may not reach the master production plan. Therefore, a secondary objective is needed to maximize the total reliability of the production plan. Let $R_{i}$ be the reliability of each unit of production, Therefore, the secondary objective is as follows,

$\max \quad \prod_{i=1}^{s}\left[1-\left(1-R_{i}\right)^{x_{i}}\right]$

subject to

$c_{i} x_{i} \leq C$ 
Problem (4) consists of one constraint which is normally associated with additional budget, $C_{i}$ is the cost of additional facility allocated to each production unit. Note that when the optimal solution of (3) is determined, there may be some slacks which make it possible to assign more facility to each production unit. Using the Lexicographic technique (Sadjadi et al. 2009), one may increase the facilities using the procedure explained by Sadjadi and Soltani (2009) in the following form,

$$
\Delta_{i}=\frac{\ln \left[1-\left(1-R_{i}\right)^{x_{i}+1}\right]-\ln \left[1-\left(1-R_{i}\right)^{x_{i}}\right]}{c_{i}} .
$$

In (5), we compute $\Delta_{i}$ for each unit and choose the maximum one. If an increase on $x_{i}$ does not violate $A x \leq b$ then we add one more unit to $X_{i}$, otherwise we examine the second largest $\Delta_{i}$ and this process continues until no increase is possible due to the violation of budget constraint or $A x \leq b$.

\section{Example 2}

Consider example (1) when there is an additional budget to assign to each five units with the budgetary and reliability data given in Table 2.

Table 2

The summary of the input data for example 2

\begin{tabular}{lllll}
\hline$R_{1}=0.90$ & $R_{2}=0.95$ & $R_{3}=0.80$ & $R_{4}=0.85$ & $R_{5}=0.90$
\end{tabular}

$c_{1}=100 \quad c_{2}=150 \quad c_{3}=180 \quad c_{4}=100 \quad c_{5}=120$

Now, consider the optimal solution with $\Gamma_{i}=0.4$ where no constraint in (4) is binding. Therefore we can use the remaining slacks to assign more facilities with a total capital budget of $C=300$. Therefore, we can make an increase only on the first, third and fourth units. Again, we calculate $\Delta_{1}, \Delta_{3}$ and $\Delta_{4}$ as follows,

$$
\begin{gathered}
\Delta_{1}=\frac{\ln \left[1-(1-0.9)^{3}\right]-\ln \left[1-(1-0.9)^{2}\right]}{100}=9.05 \times 10^{-5} \quad \Delta_{3}=\frac{\ln \left[1-(1-0.8)^{3}\right]-\ln \left[1-(1-0.8)^{2}\right]}{180}=1.82 \times 10^{-4} \\
\Delta_{4}=\frac{\ln \left[1-(1-0.85)^{4}\right]-\ln \left[1-(1-0.85)^{3}\right]}{100}=2.8743 \times 10^{-5}
\end{gathered}
$$

As we can see $\Delta_{4}>\Delta_{3}>\Delta_{1}$ and we first add one more facility to the forth unit and since any more increase could violate the constraints we terminate the process.

\section{Conclusions}

We have presented a multi-objective decision making production and reliability optimization planning. The proposed method of this paper considers a robust production plan to allocate the optimal facilities for different units of production in the first phase of the algorithm. We have also assumed that all the capacity productions for all units are subject to uncertainty in the first phase of the algorithm. The remaining slacks of the different involved constraints have been considered for the second phase of the reliability optimization. The preliminary results indicate that the proposed method of this paper could help us design better facilities.

\section{Acknowledgment}

The authors would like to thank the anonymous referees for their valuable comments and suggestions on the earlier version of this work. 


\section{References}

Ben-Tal, A., \& Nemirovski, A. (2000). Robust solutions of linear programming problems contaminated with uncertain data. Mathematical Programming, 88, 411- 421.

Bertsimas, D., \& Sim, M. (2003). Robust discrete optimization and network flows, Mathematical Programming Series B, 98, 49-71.

Kenné J P, Gharbi, A, Beit, M. (2007). Age-dependent production planning and maintenance strategies in unreliable manufacturing systems with lost sale, European Journal of Operational Research, 178, 408420.

Khademi Zare H, Fatemi Ghomi S M T, Karimi B (2006), Developing a heuristic algorithm for order production planning using network models under uncertainty conditions, Applied Mathematics and Computation, 182, 1208-1218.

Misra KB. (1972), Reliability optimization of series-parallel system, IEEE Transaction on Reliability, 21,2308.

Nikoofal, M E, Sadjadi, S. J. (2010). A robust optimization model for p-median problem with uncertain edge lengths, International Journal of Advanced Manufacturing Technology, to appear.

Ramirez-Marquez JE, Coit DW, Konak A (2004). Redundancy allocation for serie- s-parallel systems using a max-min approach, IIE Transaction, 36, 891-898.

Sadjadi, S.J., \& Omrani, H. (2008). Data envelopment analysis with uncertain data: An application for Iranian electricity distribution companies, Energy Policy, 36, 4247- 4254.

Shafia, M. A., Sadjadi, S. J. \& Jamili, A. (2010). Robust train formation, Journal of Rail and Rapid Transit, 224, 75-90.

Soyster, A. L. (1973). Convex programming with eet-inclusive constraints and applications to inexact linear programming, Operations Research, 21, 1154-1157.

Sadjadi, S J, Habibian, H \& Khaledi, V. (2009). A multi-objective decision making approach for solving quadratic multiple response surface problems, International Journal of Contemporary Mathematical Sciences, 3, 1595-1606.

Sadjadi, S J, Soltani, R, (2009). An efficient heuristic versus a robust hybrid meta-heuristic for general framework of serial-parallel redundancy problem. Reliability Engineering and System Safety, 94, 17031710. 